\title{
Neutrino Mass and the LHC
}

\section{Miha Nemevšek*}

$\dagger$

ICTP, Strada Costiera 11, Trieste, Italy and JSI, Jamova 39, 1000 Ljubljana, Slovenia

E-mail: miha@ictp.it

We discuss the possibility of probing physics behind the generation of neutrino mass and its observability at the LHC. We focus on TeV scale tree-level see-saw mechanisms and their natural origin from the minimal left-right symmetric model or grand unified theories, such as the minimal $S U(5)$. The fundamental aspect related to the Majorana nature of neutrinos is the breaking of lepton number, which can manifest itself at high energies via same-sign leptons and jets. Final states differ for different see-saw mediators and data from the LHC provide significantly improved or novel bounds on all three tree-level realisations. Simultaneously, searches for lepton number (and flavour) violation at low energies provide complementary information to colliders.

Proceedings of the Corfu Summer Institute 2012

September 8-27, 2012

Corfu, Greece

\footnotetext{
* Speaker.

${ }^{\dagger}$ I would like to thank the organisers for a pleasant and stimulating atmosphere at the workshop and summer school organised in a beautiful environment on Corfu.
} 


\section{Neutrino Mass and its Origin}

The tentative discovery of the Higgs boson [1] at the LHC paves the way to probe the origin of particle masses. Once the mass of the Higgs is determined, all its couplings are predicted [2] within the Standard Model (SM) and are proportional to the mass of the particle the boson is decaying into. This dynamical origin of mass exists for any charged fermion or gauge boson of the SM and will be scrutinised at length with current and future data.

The situation with neutrinos is quite different from that of charged fermions. We know that neutrinos have small masses [3], therefore we are led to introduce additional degrees of freedom on top of the SM ones in order to have a renormalizable theory of neutrino mass. The profound difference with respect to other fermions is that neutrinos are neutral and therefore are allowed to have a Majorana mass term [4]. If this is the case, a new mass scale associated to the origin of neutrino mass has to be introduced with its own dynamics related to lepton number breaking (see [5] for a review on new physics and [6, 7] for the LHC- $0 v 2 \beta$ connection). Probing this dynamics indirectly or directly would allow one to gain insight the origin of neutrino mass.

The theoretical framework for neutrino masses, which has been established over the years is the see-saw mechanism $[8,9,10,11,12]$, where the smallness of neutrino mass is naturally suppressed by a large scale. The size of this scale, however depends on the size of the Yukawa couplings in the new theory. If they are on the order one, like the top Yukawa in the SM, the natural scale is around $10^{14} \mathrm{GeV}$, which fits nicely with a grand unified framework [10, 11]. For smaller values, this scale might well be lower e.g. $10^{7} \mathrm{GeV}$ as in [12] or even only slightly above the electroweak scale, as imagined in $[8,9]$ if new Yukawa couplings are similar to those of charged leptons in the SM. Small Yukawa couplings are self-protected by chiral symmetry, therefore having a see-saw scale in the $\mathrm{TeV}$ energy region is a perfectly reasonable option. The issue is not the size of the Yukawa couplings, but the associated physical phenomena and our ability to predict and test them.

Here, we consider the possibility of having a theory of see-saw at $\mathrm{TeV}$ energies and discuss the potential impact the searches at LHC might have and what we already learned from current data. We focus on simple tree-level realisations and in particular on their embedding in well-defined frameworks, such as Left-Right symmetric theories and grand unified theories which actually predict a low see-saw scale in order for gauge couplings to unify. We pay special attention to lepton number violation at the LHC, describe the characteristic signals and their relation to neutrino mass origin. We review the most recent searches and point out some of the caveats which may relax (or enhance) some of the bounds. Regardless of the mechanism in question, searches by both CMS and ATLAS already provide significant improvements over the existing ones.

Theories of neutrino mass, especially when present at $\mathrm{TeV}$ scales, may relate directly to a number of phenomena at low energies. Since the successful run at the LHC is complemented by many precision measurements which probe the same underlying physics, related either to lepton number or flavour violation, we comment on this high-low energy interplay when appropriate. In some cases the sensitivity of these low energy probes match the one of the LHC, in others the very precise data test the scales beyond the reach of the LHC.

This contribution is organised as follows. In section 2, we review the salient features of the seesaw mechanism and its theoretical origin; the lepton number violation and the ability of colliders 
to test for it is discussed in section 3. Section 4 is the main part of the part of the paper, where the LHC physics is searches is presented in some detail, in particular in the context of Left-Right symmetry, type II see-saw and the minimal $S U(5)$ GUT with type III see-saw. Conclusions are left for section 5 .

\section{See-saw Mechanism}

The widely accepted framework of understanding neutrino mass goes is the so-called see-saw mechanism. It is perhaps worth remembering the origin of this framework and contrast it to the simple addition of particles with arbitrary couplings, introduced only to describe neutrino masses. It is often said that if a single mediator of neutrino mass is added to the SM, there are three types of tree-level see-saw scenarios, depending on the quantum number of the additional particle, type I for fermionic singlets, type II for bosonic triplets with $Y=2$ and type III for fermionic triplets. When one adds a heavy Majorana right-handed neutrino $v_{R}$, one can write down the following mass terms

$$
\mathscr{L}_{m}=M_{D} \bar{v}_{L} v_{R}+M_{M} v_{R}^{T} v_{R}
$$

that give a small mass to left-handed neutrinos, suppressed by the heavy Majorana mass $M_{M}$

$$
M_{v}=-M_{D}^{T} M_{M}^{-1} M_{D}
$$

However, historical development of the see-saw picture was quite different. It resulted from studies whose aim was to understand issues such as parity restoration at high scales, unification of forces and quantisation of the electric charge.

Pioneering works of $[8,9]$ connected the smallness of neutrino mass to the scale of parity restoration in the context of left-right (LR) symmetric theories. In this approach, the LR scale and therefore that of the see-saw $\left(m_{M}\right)$ is expected to be slightly above the electroweak scale. The present-day results from the LHC push this scale in the $\mathrm{TeV}$ region, but the point remains: the seesaw scale in the $\mathrm{TeV}$ region is a viable and theoretically attractive framework for small neutrino masses. Small Yukawa couplings are self-protected due to the chiral symmetry and the hierarchy between the SM is fairly small. A different approach in the context of grand unified theories was taken by $[10,11,12]$. In theories such as $S O(10)$, Dirac Yukawa couplings for the neutrino might be fairly large, similar in size to the top Yukawa, while neutrino masses are suppressed due to the large scale of unification. Despite their appeal and although a number of minimal GUT models are still viable (for a recent review, see [13]), this avenue rarely leads to any dynamics at the $\mathrm{TeV}$ scale, with some notable exceptions which we discuss below.

\section{Lepton Number}

The striking property of see-saw mechanism is breaking of lepton number resulting from the neutrino Majorana mass term. The physical consequence at low energies were studied almost immediately after the seminal work of Majorana [4] and the double nuclear beta decay without the emission of neutrinos $(0 v 2 \beta)$ was suggested $[14,15]$ to look for lepton number violation. A number of searches have been performed and experiments are currently running, that will push 

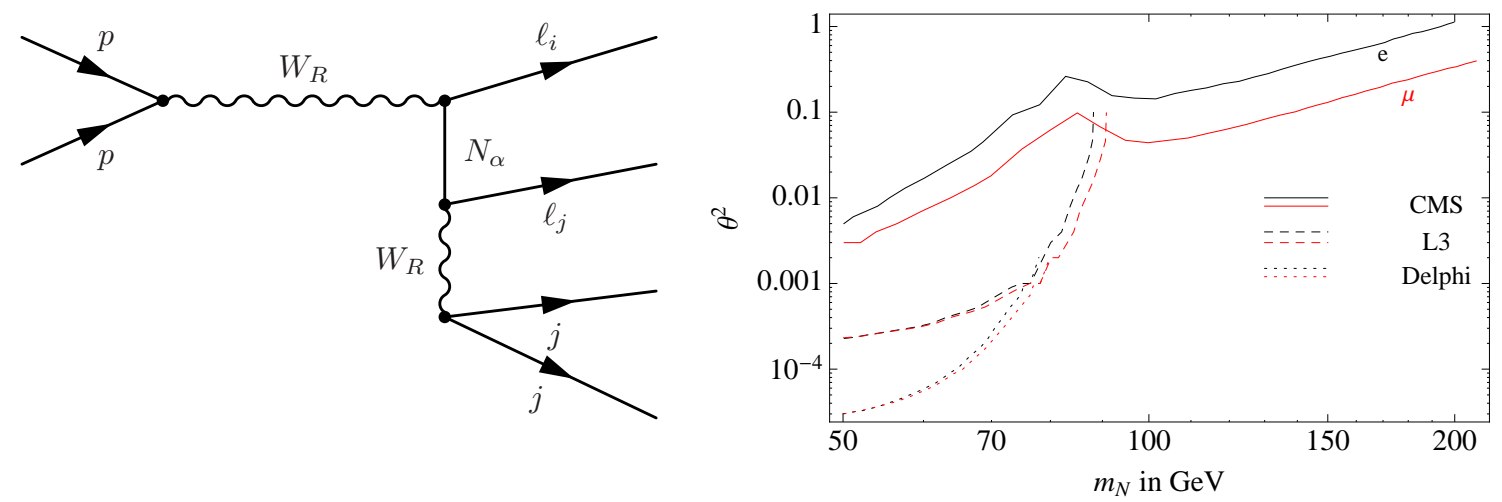

Figure 1: Left: The production of heavy Majorana neutrino at hadron colliders [20]. Right: Searches for right-handed neutrinos produced via mixing with left-handed neutrinos in the electron and muon channel at the LHC [27] and LEP [28, 29].

these limits even further. A positive signal would be of fundamental importance and the question of lepton number violation would be settled, regardless of whether the source is light Majorana neutrino exchange or new physics $[16,17]$.

While neutrino-less double beta $(0 v 2 \beta)$ search is of great importance, the real question would be what is the dynamics responsible for the Majorana mass, related either to LR symmetry or to some other physics. In order to do that, one might get more information from studies of different nuclei or by tracking the outgoing electrons [19]. However, a truly fundamental insight can only be obtained by uncovering the short-distance physics behind the neutrino mass directly at high energies. A far more transparent way than $0 v 2 \beta$ is to probe heavy neutrinos directly at hadron colliders, such as the LHC, by the KS process, proposed by [20]. This analog of neutrino-less double beta decay would allow one to obtain a remarkable insight into physics behind neutrino mass. In the following section we will discuss the physical potential and the use of this reaction at the LHC by both CMS and ATLAS collaborations.

Notice that both LHC and $0 v 2 \beta$ searches are sensitive to similar energy scales related to lepton number violation and the experimental data is coming in on similar timescales; it might well be that the first sign of LNV comes from the LHC.

\section{LHC and Neutrino Mass}

The LHC has collected a significant amount of data in the first run and performed a number of searches relevant for theories behind neutrino mass. Here, we focus mostly on the tree-level realisations, motivated by theories, such as LR and grand unified theories, such as the minimal $S U(5)$.

If neutrino mass is simply due to an addition of heavy right-handed neutrinos as in Eq. (2.2), the experimental outlook at the LHC is quite bleak. The only interactions arise from the heavylight neutrino mixing $\theta$, which couple the heavy neutrino to the SM and can produce a signal as shown in Fig. 1 (left) with a propagating $W$ [21, 22, 23, 24]. The size of this interaction is typically on the order of $\theta^{2} \sim m_{v} / m_{N}$, apart from a caveat. Namely, the Dirac mass matrix $M_{D}$ cannot be 
predicted directly from Eq. (2.2). Even if all the masses of heavy neutrinos were measured, there still remains an ambiguity, expressed in the form of an arbitrary complex matrix [25]. This allows for potentially large Yukawa couplings which cancel to give small neutrino masses. However, in this case the information on neutrino mass is lost, together with lepton number breaking [26]. Nevertheless, the searches for heavy neutrinos have been performed at the LHC [27] and limits have been set on the mixing angle, as can be seen on the right panel of Fig. 1.

\subsection{Left-Right Symmetry}

The minimal model and neutrino mass. Left-right (LR) symmetric theories were introduced [ 30 , 31] to explain the apparent violation of parity of weak interactions at low energies. Parity is postulated as an exact symmetry at high energies and is spontaneously broken $[32,33]$ to the SM gauge group by the analog of the Higgs mechanism.

The minimal LR symmetric model [8,9] is based on the $S U(2)_{L} \times S U(2)_{R} \times U(1)_{B-L}$ gauge group, augmented by LR parity that implies equality of gauge couplings $g_{L}=g_{R} \equiv g$. Quarks and leptons come in LR symmetric doublets $Q_{L, R}=(u, d)_{L, R}$ and $L_{L, R}=(v, \ell)_{L, R}$, which guarantees the presence of the right-handed neutrino and signals the presence of neutrino mass. The Higgs sector consists of a bi-doublet $\phi(2,2,0)$ and two triplets $\Delta_{L}(3,1,2), \Delta_{R}(1,3,2)$. In the first stage of symmetry breaking, the triplet develops a vacuum expectation value (vev) $v_{R}=\left\langle\Delta_{R}\right\rangle$ on the order of TeV, while $v_{L} \equiv\left\langle\Delta_{L}\right\rangle=0$. This gives a mass to the right-handed gauge boson $W_{R}$ and heavy Majorana neutrinos $N$, both on the order of $v_{R}$. In the second stage of the symmetry breaking, the bi-doublet develops a vev, which induces a small $v_{L}$ and completes the breaking to the SM, therefore giving a mass to $W_{L}$ and a Dirac mass to all the fermions. After the breaking is completed, the see-saw formula has two contributions

$$
M_{v}=-M_{D}^{T} M_{N}^{-1} M_{D}+M_{L},
$$

one due to the Dirac couplings to the SM Higgs and the other proportional to the vev of $\Delta_{L}$.

The presence of LR parity in the minimal model turns out to be of great importance, in the quark and in the leptonic sector. Here, we focus on the following possibility: $f_{L} \leftrightarrow f_{R}^{c}, \Delta_{L} \leftrightarrow$ $\Delta_{R}^{*}, \phi \rightarrow \phi^{T}$ that corresponds to charge conjugation, with the advantage that it can be gauged and embedded in a simple group such as $S O(10)$. The immediate consequence of the LR symmetry is that $g_{L}=g_{R}$ and, in order to keep the Yukawa term invariant, the Dirac mass matrices have to be symmetric. This has an immediate implication for the flavour structure of the charged current in the extended gauge sector

$$
\mathscr{L}_{q}=\frac{g}{\sqrt{2}}\left(\bar{u}_{L} V_{\text {ckm }} W_{L} d_{L}+\bar{u}_{R} V_{R}^{q} W_{R} d_{R}\right)+\text { h.c.. }
$$

Since for quarks the only source of mass for fermions is of the Dirac type, a symmetric matrix implies $V_{R}^{q}=V_{\mathrm{ckm}}^{*}$. Therefore, parity has completely fixed the flavour structure of new interactions in terms of known parameters! On one hand, this automatically guarantees the production of $W_{R}$ in proton-proton collisions, as suggested in [20] and on the other, it sets the stage for constraints from precision observables, for example from $K-\bar{K}$ mixing. Such considerations set a strong bound on the LR scale $M_{W_{R}}>1.8 \mathrm{TeV}$ [34] which has been revisited over the years [35, 36, 37] and a recent analysis arrived at $M_{W_{R}}>2.5 \mathrm{TeV}[38]$ (see also [40, 41]). 

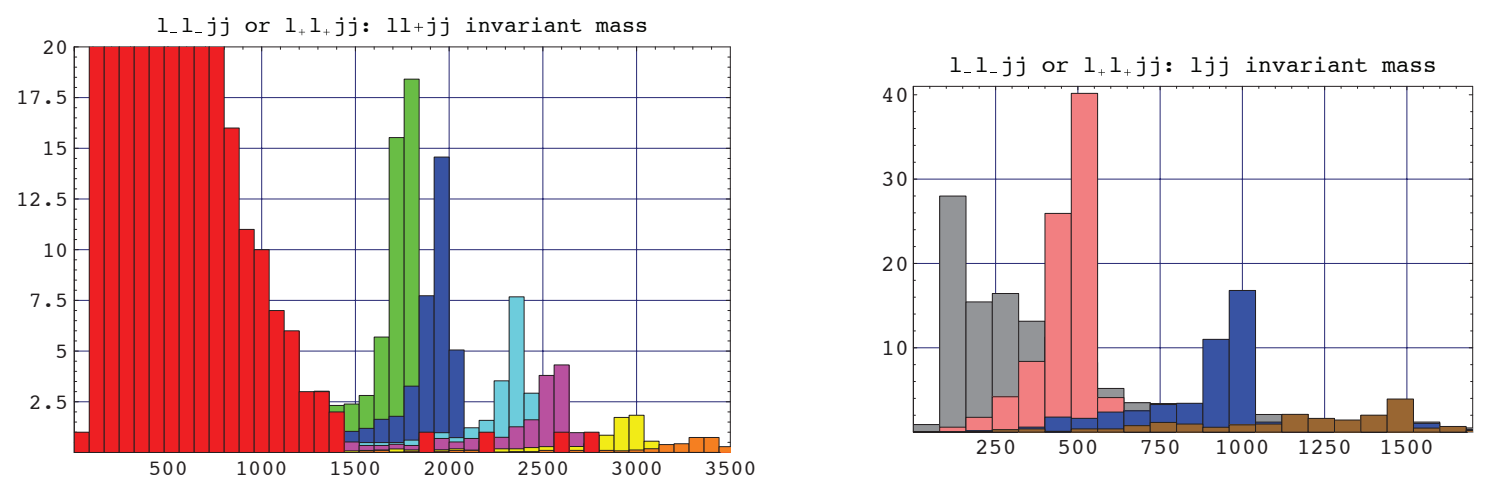

Figure 2: Left: Reconstructed peaks of $W_{R}$ mass using the invariant mass $m_{\ell \ell j j}^{\text {inv }}$ of two leptons at two jets [38]. Notice the sharp drop of the background in red once $m_{\ell \ell j j}^{\text {inv }} \gtrsim \mathrm{TeV}$. Right: Reconstructed peaks of $N$ masses using the invariant mass $m_{\ell j j}^{\text {inv }}$ of one leptons (typically highest in $p_{T}$ ) and two jets [39] with $\mathscr{L}=10 \mathrm{fb}^{-1}$.

In the leptonic sector, the situation is more involved due to the presence of both, Dirac and Majorana mass terms. Contrary to the quark sector, the leptonic charged current

$$
\mathscr{L}_{\ell}=\frac{g}{\sqrt{2}}\left(\bar{v}_{L} V_{\text {pmns }}^{\dagger} W_{L} e_{L}+\bar{N}_{R} V_{R}^{\dagger} W_{R} e_{R}\right)+\text { h.c. },
$$

allows for an arbitrary $V_{R}$, the right-handed analog of the PMNS matrix. Still, invariance under parity requires $M_{D}^{T}=M_{D}$ and $M_{L}=v_{L} / v_{R} M_{N}$ which greatly simplifies the see-saw formula and allows one to compute the Dirac mass matrix in terms of physical observables [42]

$$
M_{D}=M_{N} \sqrt{v_{L} / v_{R}-M_{N}^{-1} M_{v}}
$$

Heavy neutrino production and neutrino mass origin. Potential physical impact of the KS process [20] would be of fundamental importance for uncovering the physics responsible for the generation of neutrino mass. Heavy Majorana neutrino is produced via a Drell-Yan production of $W_{R}$, as seen in Fig. 1. Since it is of Majorana type, it decays half of the time to a lepton and the other half into an anti-lepton, giving a final state with two leptons of same or opposite sign and two jets. The final state with same-sign leptons and two jets would be a clear sign of lepton number violation and can be considered as the high-energy analog of $0 v 2 \beta$. Since there is no missing energy in the final state, the masses of three right-handed neutrinos $m_{N}$ and of $M_{W_{R}}$ can be reconstructed using the appropriate invariant mass of final state leptons and jets, producing visible peaks above the background, as shown in Fig. 2 [38]. Heavy neutrino might also decay sequentially into a lighter $N$, producing four leptons and two jets [43]. Tagging the flavour of the leptons allows one to reconstruct [44] the flavour mixing matrix $V_{R}$ in Eq. (4.3), the analog of the well-known PMNS matrix. Moreover, by studying angular distributions, the chirality of quarks [45] and leptons [46] can in principle be determined. Dedicated studies of heavy neutrino production either via charged or neutral currents were performed by ATLAS [47, 48] and CMS groups [49] which find the sensitivity of LHC to be $6(5) \mathrm{TeV}$ for $W_{R}\left(Z_{R}\right)$ mass with a luminosity of $300 \mathrm{fb}^{-1}$ running at $\sqrt{s}=14 \mathrm{TeV}$, while the heavy neutrinos can be observed with masses up to $4 \mathrm{TeV}$. 

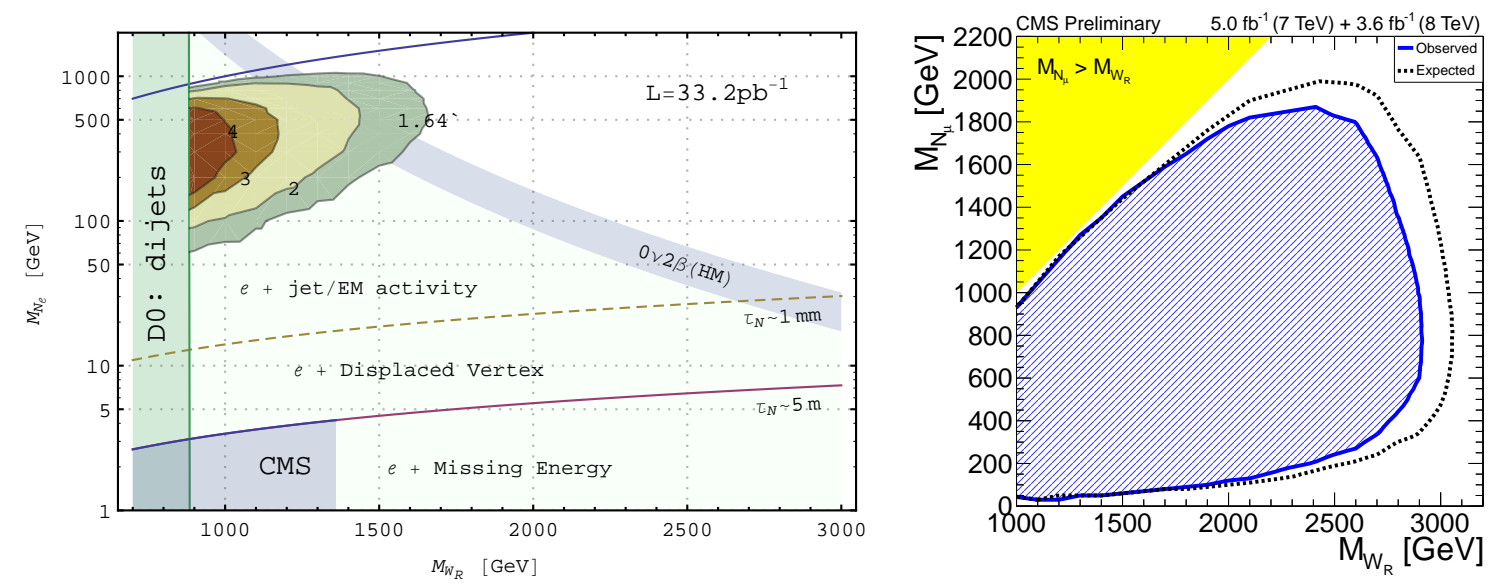

Figure 3: Left: Dependence of the final state for the heavy neutrino production as a function of $M_{W_{R}}$ and $m_{N}$, together with a rough estimate from data on lepto-quark searches. Also shown is the region corresponding to a large $0 v 2 \beta$ signal claimed by [56, 57], see [55] for details. Right: The exclusion region in the $M_{W_{R}}-m_{N}$ plane (95\% CL) in the muon channel with combined data at 7 and $8 \mathrm{TeV}$, taken from [58].

The information on the heavy neutrino mass matrix $M_{N}$ provides crucial input for establishing the exact origin of neutrino mass. The Dirac mass matrix, which governs the mixing between light and heavy neutrinos, can now be computed from Eq. (4.4). Notice that in the minimal LR model, there is no ambiguity in the determination of $M_{D}$ due to the symmetric nature of Dirac mass matrices [50, 51, 52, 53]. As a consequence, large mixings are present only in singular points and the typical size of the Dirac mass is $m_{D} \simeq \sqrt{m_{v} m_{N}}$ [42]. However, once the heavy neutrino were produced even the dominant decay channel of $N$ in Fig. 1 is suppressed by the off-shell $W_{R}$, therefore the sub-dominant decay mode via $M_{D}$ to an on-shell $W_{L}$ [54] has a chance to compete and gives a $B r \lesssim 10^{-3}$.

Searches at colliders. With the advent of the LHC finally there is data available on the energy scales beyond the long-standing bound of $M_{W_{R}}>2.5 \mathrm{TeV}$ from kaon mixing. The limits on heavy neutrinos depend on their flavour composition and on the mass of $W_{R}$. As can be seen from the left panel of Fig. 3, the signal depends on the mass of the right-handed neutrino.

In the major portion of parameter space, the neutrino will decay into two separated leptons of either same or opposite sign and two jets. The dominant background for these searches comes mostly from the production of a pair of tops and Z+jets, with QCD jets and other electroweak channels playing a minor role. Searches have been performed by both CMS [58] and ATLAS [59] and the most stringent bound at this moment is set at roughly $2.8 \mathrm{TeV}$, as shown in the right panel of Fig. 3. For generic non-zero mixing between heavy neutrinos, i.e. $V_{R} \neq 1$, the region where $m_{N}>M_{W_{R}}$ is disfavoured due to various constraints from lepton flavour violating processes [60].

If the mass of $N$ is roughly below $50 \mathrm{GeV}$, the characteristic of the signal becomes different as seen on the left panel of Fig. 3 Since the mass of $W_{R}$ is in the TeV region, fairly light decay products become boosted. This results in merging of the second lepton with the two jets and produces jet with electromagnetic activity or with a contained muon.

Although generically one expects $m_{N}$ to be on the order of LR breaking, i.e. in the TeV 
region, such light masses might be favoured, for example in order to have a warm dark matter candidate [61, 62]. For $N$ masses below few tens of $\mathrm{GeV}$, the lifetime of $N$ becomes significantly longer and at one point it produces a displaced vertex. When it is lighter than a few $\mathrm{GeV}$, it finally escapes detection and manifests as missing energy. Here, the limits from $W^{\prime} \rightarrow \ell v$ apply and one looks for a single energetic lepton and missing energy. Recent searches in this channel imply that the mass of $W_{R}$ should be bigger than $3.3 \mathrm{TeV}$ [63].

Searches that are independent of the mass of the heavy right-handed neutrino have also been performed with quarks in the final state. The di-jet searches set a limit of $M_{W_{R}}>2.3 \mathrm{TeV}$ [64], while the $W_{R} \rightarrow t \bar{b}$ channel gives $M_{W_{R}} \gtrsim 2 \mathrm{TeV}$ [65]. As in the SM, the LR group also contains a massive neutral gauge boson $Z_{L R}$, which has also been searched for [66] and the current bound on its mass stands at $M_{Z_{L R}}>\mathrm{TeV}$. Due to the fact that the $S U(2)_{R}$ gauge group is broken by a triplet Higgs, we have a mass relation $M_{Z_{L R}} \simeq 1.7 M_{W_{R}}$ and therefore in the minimal model searches for $W_{R}$ actually impose a more stringent constraint on $M_{Z_{L R}}$ than the direct searches themselves. The searches for the triplet Higgses and in particular the intricacies related to $\Delta_{L}$ signals will be discussed in the following section.

Low energy signals. Left-Right symmetry at $\mathrm{TeV}$ energies provides a potential direct insight into lepton number violation at the LHC, however at the same time it also relates to a number of low energy observables. In particular, there is a direct connection between the heavy neutrino production process [20] at the LHC and neutrino-less double beta decay, which is being searched for by a number of experiments [67].

The fact that new physics may dominate $0 v 2 \beta$ decay has been pointed out some time ago [16, 17] and LR symmetry provides a natural example [68] (see [18] for a recent review). A simple dimensional analysis, comparing the effective mass that quantifies the process mediation via light neutrinos and a generic $d=9$ operator

$$
m_{v}^{e e}=\sum_{i} V_{e i}^{2} m_{v_{i}}, \quad m_{\mathrm{np}}^{e e}=p^{2} \frac{M_{W}^{4}}{\Lambda^{5}}
$$

reveals that new physics can give a significant contribution when $\Lambda \sim \mathrm{TeV}$ ( $p \simeq 100 \mathrm{MeV})$. The short distance contribution through the exchange of heavy neutrino and $W_{R}$ can be obtained from the diagram on the left panel of Fig. 1 by replacing the protons and jets with quarks in the nucleus. Therefore, measurements at the $\operatorname{LHC}\left(M_{W_{R}}, m_{N}, V_{R}\right)$ would enable one to predict the $0 v 2 \beta$ rate $[69$, 70] with a possible large signal as long as the LR scale is in the TeV range, as seen in Fig. 4, left. ${ }^{1}$ It turns out that the contribution due to $\Delta_{R}^{++}$exchange is typically suppressed due to constraints from lepton flavour violation [69] and the same holds for the amplitudes via heavy-light neutrino mixing that are in general sub-leading [42], apart from isolated points of parameter space (see also [73]).

Another low energy signal related to $\mathrm{TeV}$ scale LR symmetry is the electric dipole moment of the electron, suppressed by a single loop in the minimal model and proportional to the Dirac mass of neutrinos [42]. Since the Dirac mass matrix can be computed from the heavy neutrino Majorana masses and mixings using Eq. (4.4), the LHC measurements relate to yet another process within the reach of future experiments as seen on the right side of Fig. 4.

\footnotetext{
${ }^{1}$ The LHC- $0 v 2 \beta$ connection $[6,7]$ is a very straightforward one in the minimal LR model. A similar relation can also be found for example in the MSSM [71] or in models with scalar di-quarks and lepto-quarks [72], however the large number of parameters of the MSSM obscures a direct correspondence between low and high energy LNV.
} 

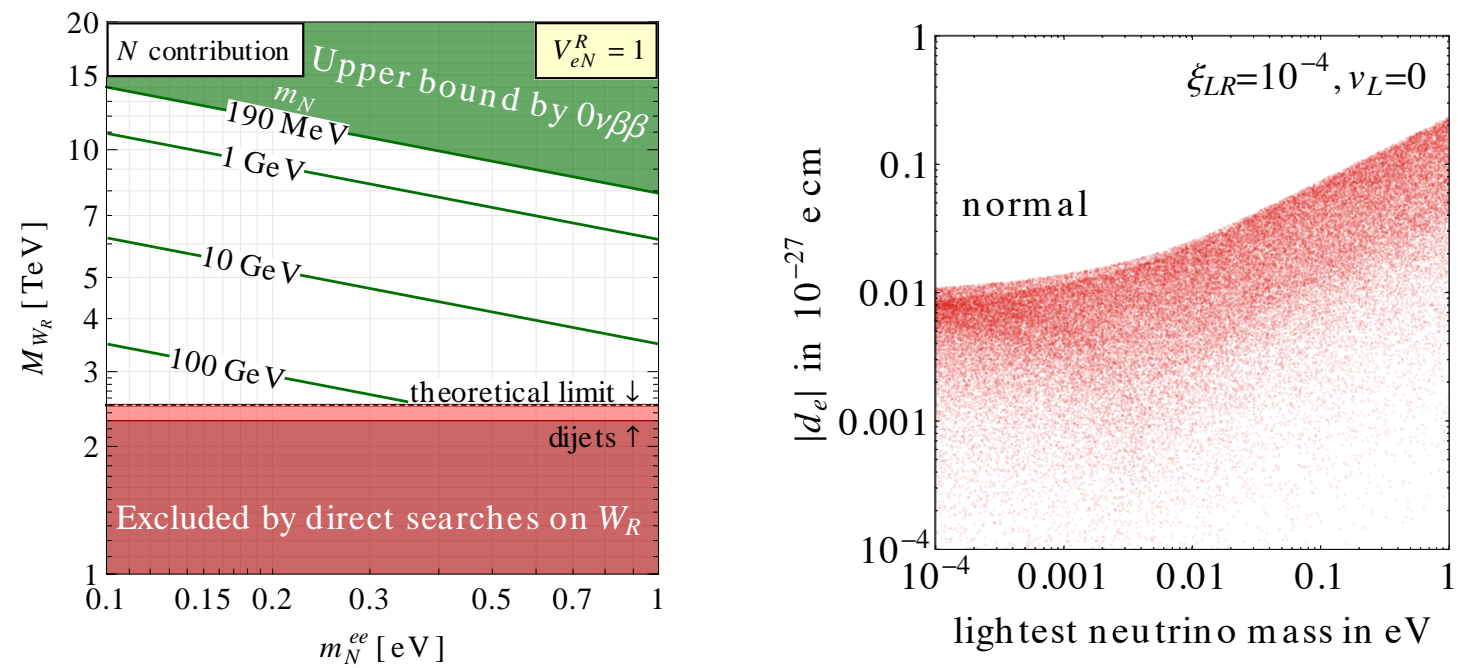

Figure 4: Left: A typical size of the LR scale as a function of the effective mass $m_{N}^{e e}$, which is probed by current experiments (see [70] for details). Right: With right handed neutrinos in the TeV range, information from the LHC provides a crucial input for the electron EDM, which is typically on the order of $10^{-28} \mathrm{e} \mathrm{cm}$ (for illustration $m_{N_{1,2,3}}=0.5,2,2.5 \mathrm{TeV}$ and $V_{R}=V_{L}^{*}$ as taken in [42]).

\subsection{Higgs triplets and neutrino mass.}

It is well-known that neutrinos can obtain a Majorana mass from a scalar triplet with $Y=2$ after it obtains a vacuum expectation value. Such representations have their natural origin in grand unified theories (GUTs), for example in $15_{H}$ of $S U(5)$ [10] (see $[74,75]$ for a minimal model with $15_{H}$ ) or in $126_{H}$ of $S O(10)[11,77]$. The weak triplet field is also contained within the Higgs sector of the minimal left-right model $[8,9]$ and gives an additional contribution to neutrino mass. Finally, one can always consider a simple scenario; an extension to the SM [78] with the triplet alone, the so-called type II see-saw case.

The weak triplet contains three complex components

$$
\Delta=\left(\begin{array}{cc}
\Delta^{+} / \sqrt{2} & \Delta^{++} \\
\Delta^{0} & -\Delta^{+} / \sqrt{2}
\end{array}\right),
$$

which can be produced pair-wise at colliders if their mass is small enough. The attractive feature of the type II scenario is a remarkable relation between the masses and mixings of light neutrinos which originates from the Majorana mass term

$$
\mathscr{L}_{m}=\frac{M_{v}}{v_{L}} L^{T} C i \sigma_{2} \Delta_{L} L+\text { h.c. }
$$

to the decay rates of the doubly charged component [79]

$$
\Gamma_{\Delta^{++} \rightarrow \ell}=\frac{m_{\Delta_{L}^{++}}}{8 \pi\left(1+\delta_{i j}\right)}\left|\frac{V_{L}^{*} m_{v} V^{\dagger}}{v_{L}}\right|_{i j}^{2},
$$

which gives a distinct signal of pairs of same-sign di-leptons. The branching ratio to different flavour of the final state leptons is sensitive to neutrino mass parameters, including the mass hierarchy and CP phases [80, 81], which provides a direct insight into the origin of neutrino mass. The 

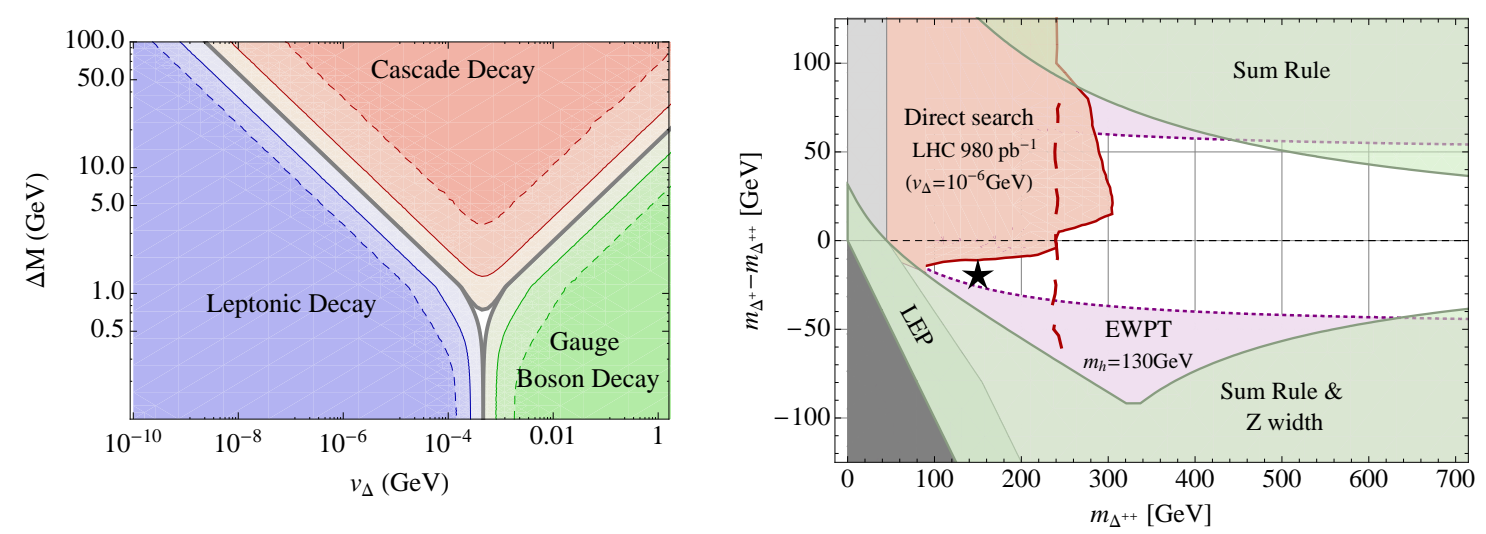

Figure 5: Left: The final state phase space diagram for the doubly charged component, depending on the vev and the mass splitting [86]. Right: An illustration of the bound on the doubly charged triplet using the actual data [90] which depends sensitively on mass splittings in agreement with electroweak precision tests [86].

components of the triplet field carry electroweak interactions, therefore they can be produced in pairs [82, 83, 84] at the LHC, single production via vector boson fusion is suppressed by the small $v_{L}$. However, the decay channel in Eq. (4.8) is not unique and the branching ratio of $\Delta^{++} \rightarrow \ell \ell$ may not be $100 \%$. For one, it depends on the size of the triplet vev. Only when $v_{L} \lesssim 10^{-4} \mathrm{GeV}$, the Yukawa couplings are large enough for this channel to dominate over the decay to two gauge bosons, governed by the gauge coupling [85]. The other important parameter which characterises the final state is the size of the mass split which is controlled by a Higgs cross-term in the potential

$$
V=V_{H}+V_{\Delta}+\left(\mu H^{T} i \sigma_{2} \Delta^{*} H+\text { h.c. }\right)+\alpha H^{\dagger} H \operatorname{Tr} \Delta^{\dagger} \Delta+\beta H^{\dagger} \Delta \Delta^{\dagger} H
$$

and is typically on the order of the electroweak scale $\Delta m^{2} \simeq \beta v^{2} / 4$.

Once the difference between the masses of the components is bigger than a few $\mathrm{GeV}$, the cascade decays of one component into another opens up [86], as seen on the left side of Fig. 5. Depending on the sign of the coupling $\beta$ that controls the mass splits, one of the components (either neutral or doubly charged) will end up as the lightest one and others will cascade into it [87]. This consideration should be taken into account when limits are set at colliders, as exemplified on the right panel of Fig. 5 since it leads to a dramatic change once the degeneracy of the masses is lifted. When the neutral component is the lightest one, it decays to neutrinos leading to a final state with missing energy, therefore the bound is weakened significantly (see also [88]).

Another region that is starting to be explored is the portion where the vev is fairly large and the decays to vector bosons dominate. The size of $v_{L}$ is controlled by the $\mu$ term in (4.9)

$$
v_{L}=\frac{\mu v^{2}}{\sqrt{2} m_{\Delta}^{2}},
$$

and is naturally suppressed by the large mass of the triplet or by the self-renormalized $\mu$ coupling. The electroweak precision data requires $v_{L}$ to be smaller than about GeV. Notice, however that the mass splitting between the components also breaks the electroweak symmetry and affects the 


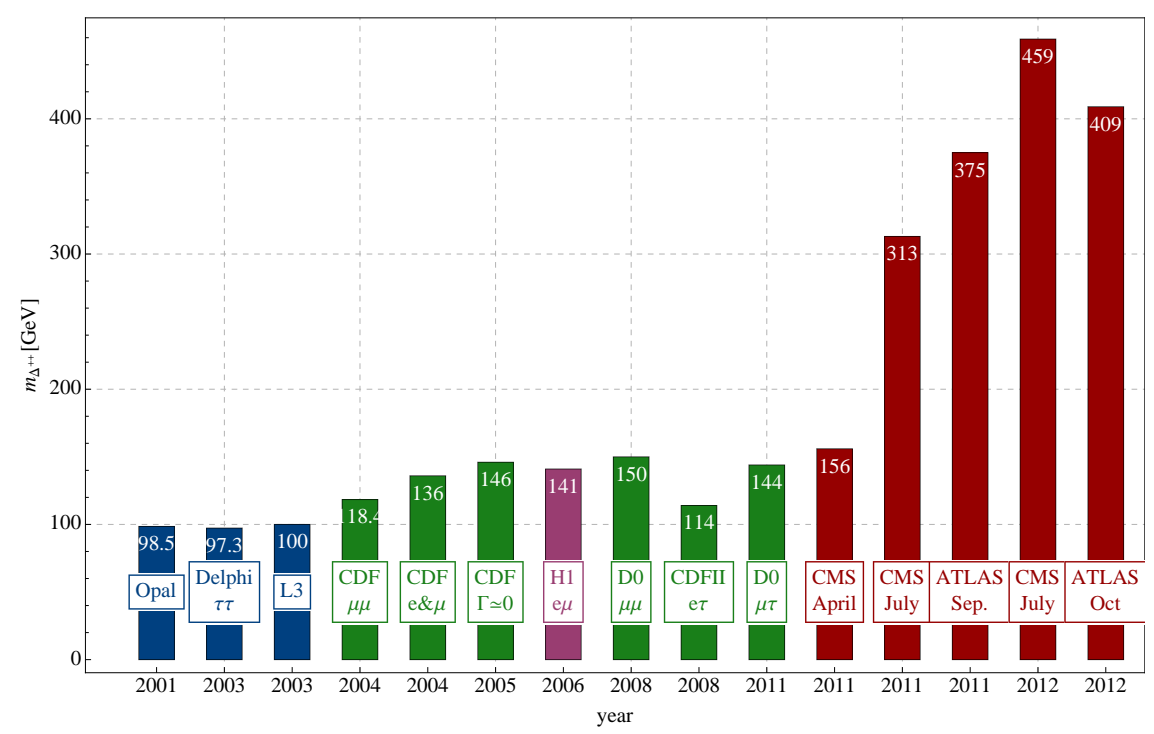

Figure 6: Development of the limits on the doubly charged Higgs of the weak triplet. The increase of beam energy at the LHC is responsible for the significant increase of the limit under the assumption of mass degeneracy of the triplet components.

precision data [86]. In particular, it contributes to the $T$ parameter with the opposite sign relative to $v_{L}$, which allows for somewhat larger $v_{L} \sim O(10 \mathrm{GeV})$, bounded by the perturbativity of $\beta$ [89]. A relatively large size of $v_{L}$ is harder to probe at the LHC due to the large SM background. Studies of vector boson fusion production were carried out [91] and sensitivity studies [92] show a possible reach of around $\mathscr{O}(100 \mathrm{GeV})$ for $v_{L} \simeq \mathrm{GeV}$. Keep in mind that in order to probe the lepton number violation in this model, both Yukawa couplings and the non-zero value for $v_{L}$ should be established, for instance in the form of same-sign tetra-leptons [93]. In any case, signals with many leptons is also a useful discrimination tool between the different see-saw models [94].

The triplet couples directly to the SM Higgs, therefore it will affect its couplings and the corresponding branching ratios, leaving the production essentially unchanged. In particular the tree-level decays to two gauge bosons, as well as the loop-induced processes, such as $h \rightarrow \gamma \gamma$ and $h \rightarrow Z \gamma$ get affected, depending on the size of the parameters $\alpha$ and $\beta$ in Eq. (4.9). The diphoton branching ratio could increase significantly if $\alpha$ is positive and large [95], or small and negative $[86,96]$. In order for this enhancement to be significant, the doubly charged component should be fairly light, below $200 \mathrm{GeV}$ or so if the potential is to be stable [97], therefore the mass splits play a significant role to allow for such small values. Together with the di-photon channel, also the $Z$-photon rate should be enhanced, as pointed out in [98]. At the same time, the lightest component should not be below $m_{h} / 2$ in order not to contradict the Higgs invisible rate.

Finally, there is another region of parameter space interesting from the low energy point of view. For small values of $v_{L}$ Yukawa couplings become large and have sizeable off-diagonal entries. For a fairly light triplet, this may lead to LFV signals [99, 101] and gives a bound [69] on

$$
v_{L} m_{\Delta^{++}} \gtrsim 100 \mathrm{eV} \mathrm{GeV} .
$$

Due to the direct connection to neutrino masses and mixings, a recent measurement of a fairly large 
$\theta_{13}$ angle ensures that cancellations due to the flavour structure cannot occur [100].

Searches at colliders. There is a standing line of direct searches for the doubly charged component of the triplet Higgs triplet. The particle has been searched for at LEP [102, 103, 104], Tevatron [105, 106, 107, 109, 110, 111], Hera [108] and finally at the LHC [90, 112]. The results are collected on Fig. 6. The bound has been steadily increasing over the years; however there is a steep improvement using the data collected at the LHC. This is due to the increase in luminosity and due to the inclusion of both pair- and associated production of all the states. There is a slight dependence on the flavour of the final states, the bounds are more significant for the $e$ and $\mu$ channel and weaker for the $\tau$. But since in type II the relation between the neutrino mixing is a direct one, a single flavour cannot dominate and the final limit is quite stable. The LHC reach for the scalar triplet is around $m_{\Delta} \simeq \mathrm{TeV}$ with the machine running at $14 \mathrm{TeV}$ [82].

Perhaps the biggest caveat in these searches is the possibility of cascade decays discussed above, which introduces another parameter, i.e. the mass splitting, which can either strengthen or weaken the limit of the degenerate case [86], as seen on the right side of Fig. 5. Notice that in the LR model, the situation is different for the right-handed triplet $\Delta_{R}$. There, the singly charged and the imaginary part of the neutral component get eaten by the right-handed gauge bosons, cascades do not occur and the bound stays at around $300 \mathrm{GeV}$ [112]. The only difference is a somewhat reduced production rate with respect to $\Delta_{L}$ due to the absence of the associated production and because of the destructive interference between the $Z$ and $\gamma^{*}$ production channel.

An experimental search in the large $v_{L}$ region is still missing. However, a re-interpretation [113] of the limits from supersymmetry searches in the same-sign di-lepton, jets and missing energy channel [114] can be done and it implies a limit of around $150 \mathrm{GeV}$ for $v_{L} \simeq \mathrm{GeV}$, again with a dependence on the sign and size of the mass splittings.

\subsection{Minimal SU(5) and type III see-saw}

Grand unified theories provide an attractive theoretical framework for understanding the origin of particle interactions, quantisation of charge and baryon number violation. The theories based on $S O(10)$ seem to be tailor-made for neutrino mass [11] since the spinorial representation $16_{F}$ necessarily contains a right-handed neutrino. However, the choice of the unifying gauge group is not truly minimal and a feasible minimal model is yet to be fully established.

On the other hand, the $S U(5)$ group still has a certain appeal to it due to the minimal possible simple group which contains the SM as its subgroup [115]. However, the minimal model with fermions in $10_{F}, \overline{5}_{F}$ and the adjoint Higgs together with $5_{H}$ is not viable in different respects:

- gauge couplings do not unify,

- neutrinos are massless,

- quark-lepton mass relations are not realistic.

Therefore, one is forced to look beyond the minimal setup for a working and hopefully predictive model. There are two possible ways to do it: either by enlarging the Higgs sector with a $15_{H}$ that gives a Majorana mass to neutrinos [10] or by introducing a fermionic adjoint $24_{F}$ [116]. The first option leads to neutrino mass via type II see-saw and requires fairly light lepto-quarks [74, 75], 


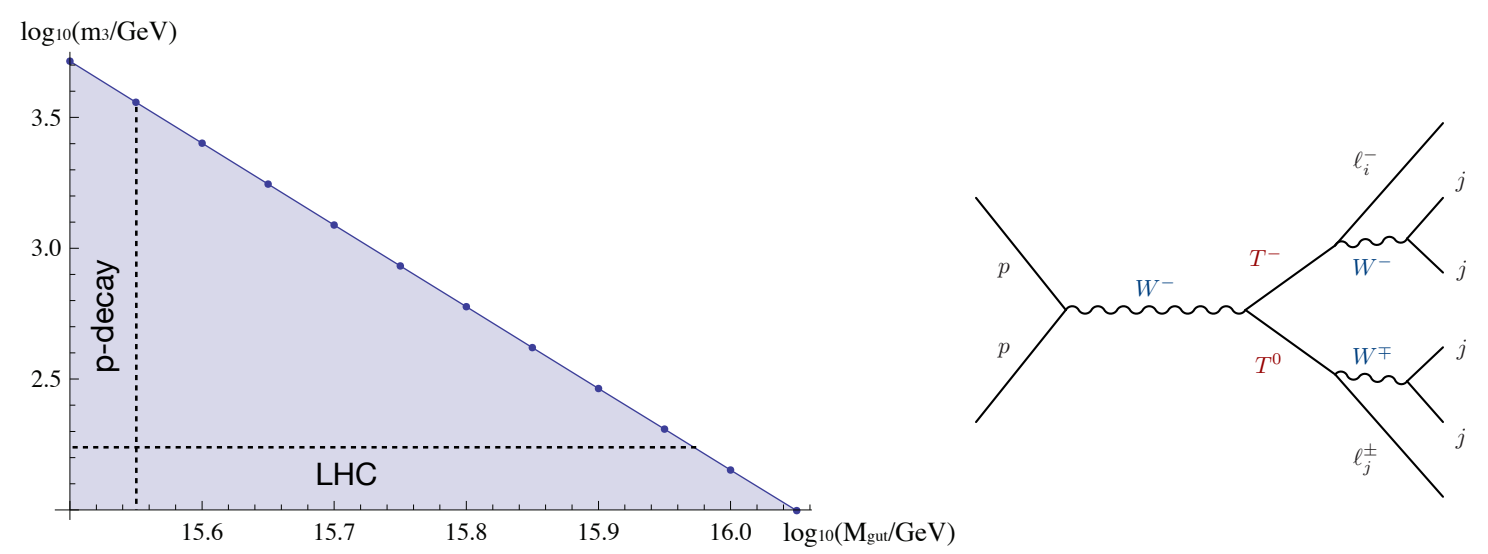

Figure 7: Left: Allowed parameter space for the triplet mass $m_{3}$ as a function of the GUT scale, following from a two loop RGE analysis [117]. Right: Associated production of triplets at the LHC and their subsequent decay to a pair of same- or opposite-sign leptons and four jets.

while the scalar triplets and therefore the possibility of testing neutrino mass origin, remain outside the reach of the LHC. The latter option, on the other hand, leads to an exciting prediction of light see-saw mediators, which could well be seen by the LHC [116, 117].

The decomposition of the adjoint of $S U(5)$ under the SM group

$$
24_{F}=(1,0)_{0}+(3,0)_{0}+(8,0)_{0}+(3,2)_{ \pm 5 / 6}
$$

reveals a fermionic singlet and a triplet with hypercharge 0 . These are tailor-made for a hybrid see-saw with a joint type I and type III [118] contribution. Since there are only two mediators, the see-saw formula is simple

$$
\left(M_{v}\right)_{i j}=\frac{v^{2}}{2}\left(\frac{y_{i}^{T} y_{j}^{T}}{m_{T}}+\frac{y_{i}^{S} y_{j}^{S}}{m_{S}}\right),
$$

where $m_{T, S}$ are the masses of the triplet and the singlet with their corresponding Yukawa couplings $y_{T, S}$. The neutrino mass matrix in Eq. (4.13) has rank two, therefore the model predicts the lightest neutrino mass to be massless, up to two loop corrections.

The attractive feature of this model is the prediction of a light triplet from gauge coupling unification which works in the following way. The threshold effects in RGE running from heavy states in the adjoint, fermionic and those remaining Higgses from $24_{H}$, change the running of $\alpha_{2}$ and leave $\alpha_{1,3}$ intact, at least at the one loop level. In the SM, the $\alpha_{1}$ and $\alpha_{2}$ meet at around $10^{13} \mathrm{GeV}$, but if triplets are light they meet at a high scale of $10^{16} \mathrm{GeV}$. In order for $\alpha_{3}$ to catch up as well, the octets in (4.12) should lie below the GUT scale, too. If the effect of all the states in Eq. (4.12) is properly taken into account, unification can be achieved and a unique solution for the spectrum emerges

$$
m_{3} \lesssim 10^{3} \mathrm{GeV}, \quad m_{8} \simeq 10^{8} \mathrm{GeV}, \quad m_{3,2} \lesssim M_{G U T} .
$$

Here, $m_{3,8}=\left(m_{3 F}^{4} m_{3 B}\right)^{1 / 5}$ is the effective mass since both fermions and bosons are running in the loops with different coefficients. A simple one-loop [116] estimate shows that the triplet has to be very light, and that its mass is anti-correlated with the scale of unification. This conclusion remains at two loops [117] (left side of Fig. 7) for somewhat larger triplet masses than the one 
loop result would suggest. Recently, an improved study [119] confirms this prediction with a three loop calculation, which is within the uncertainty of gauge coupling measurements of the two-loop result. As seen on the left side of Fig. 6, the combined limits from proton decay searches and the direct search at the LHC can either discover or rule out this minimal version of $S U(5)$.

Type III at LHC. The attractive feature of this minimal model is that it predicts a low scale of see-saw from the minimal grand unified theory. Moreover, the mediator carries gauge interactions, therefore its components can be produced pair-wise at the LHC [120, 121, 94, 122, 123, 124] as shown on the right side of Fig. 7.

Once the triplet would be produced on-shell, one would like to verify whether it is in fact the particle responsible for neutrino mass generation. In the absence of Yukawa couplings $y_{T, S}$ the triplet would decouple from the SM and become stable if its components were degenerate in mass. Even though their masses are equal at tree-level, they get split by electroweak corrections [117] at one loop and the neutral component becomes lighter by about $160 \mathrm{MeV}$. This opens up the channel $T^{+} \rightarrow T^{0} \pi^{+}$, which is not sensitive to Yukawa couplings and the relation to neutrino mass might be lost. However, once the Yukawa couplings needed for neutrino masses via Eq. (4.13) are introduced, both charged and neutral components decay to a charged lepton and a SM gauge boson, as shown in Fig. 6, right. The branching ratios to different flavours probes the neutrino mass matrix [116] and is sensitive to the hierarchy and CP phases [123].

Since formula (4.13) is a special case of (2.2), it suffers from the same ambiguity and the Yukawa couplings can become large. In such case, lepton flavour violating processes set an upper limit on their size. The most stringent process is set by $\mu-e$ conversion [126] which proceeds at tree-level. This happens because the same Yukawa couplings that generate neutrino masses, enter the off-diagonal term in the charged lepton mass matrix and generate non-universal couplings to the $Z$ boson.

Together with the fermionic triplet, the model also predicts a light bosonic counterpart, coming from $24_{H}$. It couples to the Higgs boson, similar to the case of type II see-saw above and it can have an impact on the Higgs decay rate. In particular if its mass is around $100 \mathrm{GeV}$, still allowed by the LEP data, it can easily increase the di-photon rate and also the $Z \gamma$ channel [127].

Recent data from the LHC was used to search for the heavy fermions of the type III see-saw model. The production channel on Fig. 7 with two isolated leptons of equal or opposite charge, together with four jets was used by both, CMS [128] and ATLAS [129] collaborations to search for the heavy leptons. Depending on the flavour of the final state SM leptons, the limit varies from $180-200 \mathrm{GeV}$ [128] and even to $245 \mathrm{GeV}$ [129]. With the LHC running at $\sqrt{s}=10 \mathrm{TeV}$ and with $10(100) \mathrm{fb}^{-1}$ of data collected, the reach for the triplet mass is around $450(700) \mathrm{GeV}$ [123].

\section{Conclusions}

The era of the LHC has begun and the discovery of the Higgs boson allows us to get an insight into the origin of particle masses. The most obvious call for new physics is the need to understand the origin of neutrino mass, which the SM predicted to be massless. Remarkably enough, LHC has the potential to probe such physics if the scale is around $\mathrm{TeV}$ by giving a direct insight into lepton number breaking. 
The discovery potential for singlet fermions is quite weak from the theory perspective. Its observation would require fine-tuning of Yukawa couplings, contrary to the spirit of see-saw. Nevertheless, the limits from the LHC extend the previous LEP searches above $100 \mathrm{GeV}$, albeit for relatively large mixings of $10 \%$.

The situation is completely different for the minimal LR symmetric model, the original theory that led to the see-saw mechanism. It offers rich phenomenology, both at colliders and low-energy experiments. The LHC is tailor-made for probing the heavy neutrino via the KS reaction [20], with a discovery potential up to $M_{W_{R}} \simeq 6 \mathrm{TeV}$ in the late stage of the running. Information on the heavy neutrino mass matrix then allows to predict the Dirac couplings and connect directly to a number of low energy processes, such as $0 v 2 \beta$, LFV decays and the electron edm.

The triplet Higgs, either as a light remnant of LR or $S U(5)$, or when considered as a scenario in its own right, has a realistic chance of being observed at the LHC. The characteristic signature of a doubly charged scalar decaying into same-sign leptons has been explored and limits at around $400 \mathrm{GeV}$ now exist for components degenerate in mass and may relax significantly when splittings are taken into account.

Although LR theories and type II see-saw can be embedded in grand unified frameworks, their scale is not necessarily low. On the contrary, the minimal $S U(5)$ based on an extension with a single adjoint fermionic representation predicts a low scale for type III see-saw, well within the reach of the LHC. It too contains a source of lepton number breaking, this time with two leptons and four isolated jets. The LHC already excludes part of the allowed parameter space in this minimal setup, and with future proton decay searches such as Hyper-K [130], this model is either to be found or completely excluded, a feature not commonly with many other frameworks.

Acknowledgments. I would like to acknowledge and thank my collaborators Borut Bajc, Alessio Maiezza, Alejandra Melfo, Fabrizio Nesti, Goran Senjanović, Francesco Vissani, Vladimir Tello and Yue Zhang for numerous discussions and collaboration. Special thanks are to Fabrizio Nesti, Goran Senjanović and Vladimir Tello for a careful reading of the manuscript and many indispensable suggestions.

\section{References}

[1] P. W. Higgs, Phys. Rev. Lett. 13 (1964) 508. P. W. Higgs, Phys. Rev. 145 (1966) 1156.

[2] S. Weinberg, Phys. Rev. Lett. 19 (1967) 1264.

[3] A. Strumia and F. Vissani, hep-ph/0606054.

[4] E. Majorana, Nuovo Cim. 14 (1937) 171.

[5] R. N. Mohapatra and A. Y. Smirnov, Ann. Rev. Nucl. Part. Sci. 56 (2006) 569 [hep-ph/0603118].

[6] G. Senjanović, J. Phys. Conf. Ser. 136 (2008) 022039 [arXiv:0911.0029 [hep-ph]].

[7] G. Senjanović, Int. J. Mod. Phys. A 26 (2011) 1469 [arXiv:1012.4104 [hep-ph]].

[8] P. Minkowski, Phys. Lett. B 67 (1977) 421.

[9] R. N. Mohapatra and G. Senjanović, Phys. Rev. Lett. 44 (1980) 912.

[10] S. L. Glashow, NATO Adv. Study Inst. Ser. B Phys. 59 (1980) 687. 
[11] M. Gell-Mann, P. Ramond and R. Slansky, Conf. Proc. C 790927 (1979) 315.

[12] T. Yanagida, Conf. Proc. C 7902131 (1979) 95.

[13] G. Senjanović, AIP Conf. Proc. 1200 (2010) 131 [arXiv:0912.5375 [hep-ph]].

[14] G. Racah, N.Cim. 14 (1937) 322;

[15] W.H. Furry, Phys. Rev. 56 (1939) 1184.

[16] G. Feinberg, M. Goldhaber, Proc. Nat. Ac. Sci. USA 45 (1959) 1301;

[17] B. Pontecorvo, Phys. Lett. B 26 (1968) 630.

[18] W. Rodejohann, Int. J. Mod. Phys. E 20 (2011) 1833 [arXiv:1106.1334 [hep-ph]].

[19] R. Arnold et al. [SuperNEMO Collaboration], Eur. Phys. J. C 70 (2010) 927 [arXiv:1005.1241 [hep-ex]].

[20] W. -Y. Keung and G. Senjanović, Phys. Rev. Lett. 50 (1983) 1427.

[21] A. Datta, M. Guchait and A. Pilaftsis, Phys. Rev. D 50 (1994) 3195 [hep-ph/9311257].

[22] A. Datta, M. Guchait and D. P. Roy, Phys. Rev. D 47 (1993) 961 [hep-ph/9208228].

[23] T. Han and B. Zhang, Phys. Rev. Lett. 97 (2006) 171804 [hep-ph/0604064].

[24] F. del Aguila, J. A. Aguilar-Saavedra and R. Pittau, JHEP 0710 (2007) 047 [hep-ph/0703261].

[25] J. A. Casas and A. Ibarra, Nucl. Phys. B 618 (2001) 171 [hep-ph/0103065].

[26] J. Kersten and A. Y. .Smirnov, Phys. Rev. D 76 (2007) 073005 [arXiv:0705.3221 [hep-ph]].

[27] S. Chatrchyan et al. [CMS Collaboration], Phys. Lett. B 717 (2012) 109 [arXiv:1207.6079 [hep-ex]].

[28] P. Abreu et al. [DELPHI Collaboration], Z. Phys. C 74 (1997) 57 [Erratum-ibid. C 75 (1997) 580].

[29] O. Adriani et al. [L3 Collaboration], Phys. Lett. B 295 (1992) 371.

[30] J. C. Pati and A. Salam, Phys. Rev. D 10 (1974) 275 [Erratum-ibid. D 11 (1975) 703].

[31] R. N. Mohapatra and J. C. Pati, Phys. Rev. D 11 (1975) 2558.

[32] G. Senjanović, R. Mohapatra, Phys. Rev. D 12 (1975) 1502.

[33] G. Senjanović, Nucl. Phys. B 153 (1979) 334.

[34] G. Beall, M. Bander and A. Soni, Phys. Rev. Lett. 48 (1982) 848.

[35] R. N. Mohapatra, G. Senjanović and M. D. Tran, Phys. Rev. D 28 (1983) 546.

[36] K. Kiers, J. Kolb, J. Lee, A. Soni and G. -H. Wu, Phys. Rev. D 66 (2002) 095002 [hep-ph/0205082].

[37] Y. Zhang, H. An, X. Ji and R. N. Mohapatra, Phys. Rev. D 76 (2007) 091301 [arXiv:0704.1662 [hep-ph]].

[38] A. Maiezza, M. Nemevšek, F. Nesti and G. Senjanović, Phys. Rev. D 82 (2010) 055022 [arXiv:1005.5160 [hep-ph]].

[39] Courtesy of F. Nesti.

[40] For a stringent bound on the heavy doublet in the Higgs bi-doublet, see M. Blanke, A. J. Buras, K. Gemmler and T. Heidsieck, JHEP 1203 (2012) 024 [arXiv:1111.5014 [hep-ph]]. 
[41] S. Bertolini, J. O. Eeg, A. Maiezza and F. Nesti, Phys. Rev. D 86 (2012) 095013 [arXiv:1206.0668 [hep-ph]].

[42] M. Nemevšek, G. Senjanović and V. Tello, Phys. Rev. Lett. 110 (2013) 151802 arXiv:1211.2837 [hep-ph].

[43] C. -Y. Chen and P. S. B. Dev, Phys. Rev. D 85 (2012) 093018 [arXiv:1112.6419 [hep-ph]].

[44] J. A. Aguilar-Saavedra, F. Deppisch, O. Kittel and J. W. F. Valle, Phys. Rev. D 85 (2012) 091301 [arXiv:1203.5998 [hep-ph]].

[45] S. Gopalakrishna, T. Han, I. Lewis, Z. -g. Si and Y. -F. Zhou, Phys. Rev. D 82 (2010) 115020 [arXiv:1008.3508 [hep-ph]].

[46] T. Han, I. Lewis, R. Ruiz and Z. -g. Si, Phys. Rev. D 87 (2013) 035011 [arXiv:1211.6447 [hep-ph]].

[47] A. Ferrari, J. Collot, M-L. Andrieux, B. Belhorma, P. de Saintignon, J-Y. Hostachy, P. .Martin and M. Wielers, Phys. Rev. D 62 (2000) 013001.

[48] A. Ferrari and J. Collot, ATL-PHYS-2000-034.

[49] S. N. Gninenko, M. M. Kirsanov, N. V. Krasnikov and V. A. Matveev, Phys. Atom. Nucl. 70 (2007) 441.

[50] D. Falcone, Phys. Rev. D 68 (2003) 033002 [hep-ph/0305229].

[51] E. K. Akhmedov and M. Frigerio, Phys. Rev. Lett. 96 (2006) 061802 [hep-ph/0509299].

[52] P. Hosteins, S. Lavignac and C. A. Savoy, Nucl. Phys. B 755 (2006) 137 [hep-ph/0606078].

[53] E. K. Akhmedov and M. Frigerio, JHEP 0701 (2007) 043 [hep-ph/0609046].

[54] F. del Aguila, J. A. Aguilar-Saavedra and J. de Blas, PoS ICHEP 2010 (2010) 296 [arXiv:1012.1327 [hep-ph]].

[55] M. Nemevšek, F. Nesti, G. Senjanović and Y. Zhang, Phys. Rev. D 83 (2011) 115014 [arXiv:1103.1627 [hep-ph]].

[56] H.V. Klapdor-Kleingrothaus et al., Phys. Lett. B586 (2004) 198;

[57] H.V. Klapdor-Kleingrothaus, I.V. Krivosheina, Mod. Phys. Lett. A21 (2006) 1547.

[58] [CMS Collaboration], CMS-PAS-EXO-11-002. CMS-PAS-EXO-12-004. CMS-PAS-EXO-12-017.

[59] [ATLAS Collaboration], ATLAS-CONF-2011-115. ATLAS-CONF-2012-139.

[60] V. Cirigliano, A. Kurylov, M. J. Ramsey-Musolf and P. Vogel, Phys. Rev. D 70 (2004) 075007 [hep-ph/0404233].

[61] F. Bezrukov, H. Hettmansperger and M. Lindner, Phys. Rev. D 81 (2010) 085032 [arXiv:0912.4415 [hep-ph]].

[62] M. Nemevšek, G. Senjanović and Y. Zhang, JCAP 1207 (2012) 006 [arXiv:1205.0844 [hep-ph]].

[63] S. Chatrchyan et al. [CMS Collaboration], arXiv:1302.2812 [hep-ex]. CMS-PAS-EXO-12-010. CMS-PAS-EXO-12-060.

[64] G. Aad et al. [ATLAS Collaboration], Eur. Phys. J. C 72 (2012) 2241 [arXiv:1209.4446 [hep-ex]]. JHEP 1301 (2013) 029 [arXiv:1210.1718 [hep-ex]]. [CMS Collaboration], CMS-PAS-EXO-12-059.

[65] [CMS Collaboration], CMS-PAS-B2G-12-010. 
[66] S. Chatrchyan et al. [CMS Collaboration], Phys. Lett. B 720 (2013) 63 [arXiv:1212.6175 [hep-ex]].

[67] J. J. Gomez-Cadenas, J. Martin-Albo, M. Mezzetto, F. Monrabal and M. Sorel, Riv. Nuovo Cim. 35 (2012) 29 [arXiv:1109.5515 [hep-ex]].

[68] R. N. Mohapatra and G. Senjanović, Phys. Rev. D 23 (1981) 165.

[69] V. Tello, M. Nemevšek, F. Nesti, G. Senjanović and F. Vissani, Phys. Rev. Lett. 106 (2011) 151801 [arXiv:1011.3522 [hep-ph]].

[70] M. Nemevšek, F. Nesti, G. Senjanović and V. Tello, arXiv:1112.3061 [hep-ph].

[71] B. C. Allanach, C. H. Kom and H. Pas, Phys. Rev. Lett. 103 (2009) 091801 [arXiv:0902.4697 [hep-ph]].

[72] J. C. Helo, M. Hirsch, S. G. Kovalenko and H. Pas, arXiv:1303.0899 [hep-ph].

[73] J. Barry and W. Rodejohann, arXiv:1303.6324 [hep-ph].

[74] I. Dorsner and P. Fileviez Perez, Nucl. Phys. B 723 (2005) 53 [hep-ph/0504276].

[75] I. Dorsner, P. Fileviez Perez and R. Gonzalez Felipe, Nucl. Phys. B 747 (2006) 312 [hep-ph/0512068].

[76] M. Magg and C. Wetterich, Phys. Lett. B 94 (1980) 61.

[77] G. Lazarides, Q. Shafi and C. Wetterich, Nucl. Phys. B 181 (1981) 287.

[78] T. P. Cheng and L. -F. Li, Phys. Rev. D 22 (1980) 2860.

[79] E. J. Chun, K. Y. Lee and S. C. Park, Phys. Lett. B 566 (2003) 142 [hep-ph/0304069].

[80] J. Garayoa and T. Schwetz, JHEP 0803 (2008) 009 [arXiv:0712.1453 [hep-ph]].

[81] M. Kadastik, M. Raidal and L. Rebane, Phys. Rev. D 77 (2008) 115023 [arXiv:0712.3912 [hep-ph]].

[82] G. Azuelos, K. Benslama, J. Ferland, J. Phys. G 32 (2006) 73; [arXiv:hep-ph/0503096].

[83] T. Han, B. Mukhopadhyaya, Z. Si, K. Wang, Phys. Rev. D 76 (2007) 075013;

[84] A.G. Akeroyd, M. Aoki, H. Sugiyama, Phys. Rev. D 77 (2008) 075010. [arXiv:0712.4019 [hep-ph]].

[85] P. Fileviez Perez, T. Han, G.-y. Huang, T. Li, K. Wang, Phys. Rev. D78 (2008) 015018.

[86] A. Melfo, M. Nemevšek, F. Nesti, G. Senjanović and Y. Zhang, Phys. Rev. D 85 (2012) 055018 [arXiv:1108.4416 [hep-ph]].

[87] A. G. Akeroyd and H. Sugiyama, Phys. Rev. D 84 (2011) 035010 [arXiv:1105.2209 [hep-ph]].

[88] M. Aoki, S. Kanemura and K. Yagyu, Phys. Rev. D 85 (2012) 055007 [arXiv:1110.4625 [hep-ph]].

[89] S. Kanemura and K. Yagyu, Phys. Rev. D 85 (2012) 115009 [arXiv:1201.6287 [hep-ph]].

[90] S. Chatrchyan et al. [CMS Collaboration], Eur. Phys. J. C 72 (2012) 2189 [arXiv:1207.2666 [hep-ex]]. CMS-PAS-HIG-11-001, CMS-PAS-HIG-11-001, CMS-PAS-HIG-12-005.

[91] S. Godfrey and K. Moats, Phys. Rev. D 81 (2010) 075026 [arXiv:1003.3033 [hep-ph]].

[92] C. -W. Chiang, T. Nomura and K. Tsumura, Phys. Rev. D 85 (2012) 095023 [arXiv:1202.2014 [hep-ph]].

[93] E. J. Chun and P. Sharma, arXiv:1304.5059 [hep-ph].

[94] F. del Aguila and J. A. Aguilar-Saavedra, Nucl. Phys. B 813 (2009) 22 [arXiv:0808.2468 [hep-ph]]. 
[95] A. Arhrib, R. Benbrik, M. Chabab, G. Moultaka and L. Rahili, JHEP 1204 (2012) 136 [arXiv:1112.5453 [hep-ph]].

[96] A. G. Akeroyd and S. Moretti, Phys. Rev. D 86 (2012) 035015 [arXiv:1206.0535 [hep-ph]].

[97] E. J. Chun, H. M. Lee and P. Sharma, JHEP 1211 (2012) 106 [arXiv:1209.1303 [hep-ph]].

[98] C. -S. Chen, C. -Q. Geng, D. Huang and L. -H. Tsai, arXiv:1302.0502 [hep-ph].

[99] A.G. Akeroyd, M. Aoki, H. Sugiyama, Phys. Rev. D79 (2009) 113010;

[100] J. Chakrabortty, P. Ghosh and W. Rodejohann, Phys. Rev. D 86 (2012) 075020 [arXiv:1204.1000 [hep-ph]].

[101] T. Fukuyama, H. Sugiyama and K. Tsumura, JHEP 1003 (2010) 044 [arXiv:0909.4943 [hep-ph]].

[102] G. Abbiendi et al. [ OPAL Collaboration ], Phys. Lett. B526 (2002) 221-232. [hep-ex/0111059].

[103] J. Abdallah et al. [ DELPHI Collaboration ], Phys. Lett. B552 (2003) 127-137. [hep-ex/0303026].

[104] P. Achard et al. [ L3 Collaboration ], Phys. Lett. B576 (2003) 18-28. [hep-ex/0309076].

[105] V. M. Abazov et al. [ D0 Collaboration ], Phys. Rev. Lett. 93 (2004) 141801. [hep-ex/0404015].

[106] D. E. Acosta et al. [ CDF Collaboration ], Phys. Rev. Lett. 93 (2004) 221802. [hep-ex/0406073].

[107] D. E. Acosta et al. [ CDF Collaboration ], Phys. Rev. Lett. 95 (2005) 071801. [hep-ex/0503004].

[108] A. Aktas et al. [ H1 Collaboration ], Phys. Lett. B638 (2006) 432-440. [hep-ex/0604027].

[109] V. M. Abazov et al. [ D0 Collaboration ], Phys. Rev. Lett. 101 (2008) 071803. [arXiv:0803.1534 [hep-ex]].

[110] T. Aaltonen et al. [ The CDF Collaboration ], Phys. Rev. Lett. 101 (2008) 121801. [arXiv:0808.2161 [hep-ex]].

[111] V. M. Abazov et al. [ D0 Collaboration ], [arXiv:1106.4250 [hep-ex]].

[112] G. Aad et al. [ATLAS Collaboration], Eur. Phys. J. C 72 (2012) 2244 [arXiv:1210.5070 [hep-ex]]. Phys. Rev. D 85 (2012) 032004 [arXiv:1201.1091 [hep-ex]].

[113] Y. Zhang, private communication.

[114] S. Chatrchyan et al. [CMS Collaboration], Phys. Rev. Lett. 109 (2012) 071803 [arXiv:1205.6615 [hep-ex]].

[115] H. Georgi and S. L. Glashow, Phys. Rev. Lett. 32 (1974) 438.

[116] B. Bajc and G. Senjanović, JHEP 0708 (2007) 014 [hep-ph/0612029].

[117] B. Bajc, M. Nemevšek and G. Senjanović, Phys. Rev. D 76 (2007) 055011 [hep-ph/0703080].

[118] R. Foot, H. Lew, X. G. He and G. C. Joshi, Z. Phys. C 44 (1989) 441.

[119] L. di Luzio, talks at BLV 2013 and Portorož 2013.

[120] E. Ma and D. P. Roy, Nucl. Phys. B 644 (2002) 290 [hep-ph/0206150].

[121] R. Franceschini, T. Hambye and A. Strumia, Phys. Rev. D 78 (2008) 033002 [arXiv:0805.1613 [hep-ph]].

[122] F. del Aguila and J. A. Aguilar-Saavedra, Phys. Lett. B 672 (2009) 158 [arXiv:0809.2096 [hep-ph]]. 
[123] A. Arhrib, B. Bajc, D. K. Ghosh, T. Han, G. -Y. Huang, I. Puljak and G. Senjanović, Phys. Rev. D 82 (2010) 053004 [arXiv:0904.2390 [hep-ph]].

[124] T. Li and X. -G. He, Phys. Rev. D 80 (2009) 093003 [arXiv:0907.4193 [hep-ph]].

[125] X. -G. He and S. Oh, JHEP 0909 (2009) 027 [arXiv:0902.4082 [hep-ph]].

[126] J. F. Kamenik and M. Nemevšek, JHEP 0911 (2009) 023 [arXiv:0908.3451 [hep-ph]].

[127] C. -S. Chen, C. -Q. Geng, D. Huang and L. -H. Tsai, arXiv:1301.4694 [hep-ph].

[128] S. Chatrchyan et al. [CMS Collaboration], Phys. Lett. B 718 (2012) 348 [arXiv:1210.1797 [hep-ex]].

[129] [ATLAS Collaboration], ATLAS-CONF-2013-019.

[130] K. Abe, T. Abe, H. Aihara, Y. Fukuda, Y. Hayato, K. Huang, A. K. Ichikawa and M. Ikeda et al., arXiv:1109.3262 [hep-ex]. 